\title{
RT-QUiC in multiple system atrophy: the biomarker of the future? and other updates on recent autonomic research
}

\author{
Mitchell G. Miglis ${ }^{1,2} \cdot$ Nicholas Larsen $^{1} \cdot$ Srikanth Muppidi ${ }^{1}$
}

Received: 2 January 2021 / Accepted: 4 January 2021 / Published online: 29 January 2021

(c) Springer-Verlag GmbH Germany, part of Springer Nature 2021

Keywords Multiple system atrophy $\cdot$ Alpha-synuclein $\cdot$ Postural tachycardia syndrome

\section{Protein misfolding cyclic amplification in multiple system atrophy: The biomarker of the future?}

Distinguishing Parkinson's disease (PD) from multiple system atrophy (MSA) can be quite challenging for clinicians, a topic we have covered in several prior updates. Part of this difficulty lies in the lack of sensitive and specific biomarkers to help differentiate subtypes of the $\alpha$-synucleinopathies. Recently, the technique of protein misfolding cyclic amplification (PMCA), also referred to as real-time quakinginduced conversion (RT-QuIC) when used in the synucleinopathies, has been developed as a new potential biomarker. PMCA utilizes the concept of seeding-nucleation, whereby initial seeds of $\alpha$-synuclein recruit other soluble monomers to form insoluble aggregates, thereby cyclically amplifying the aggregation to acceptable levels that can be detected in biofluids, such as blood as cerebrospinal fluid (CSF).

In their article, "Discriminating $\alpha$-synuclein strains in Parkinson's disease and multiple system atrophy, [1]" Shahnawaz et al. utilized PMCA to amplify CSF of 94 patients with PD, 75 with MSA (with similar percentages of MSA-P and MSA-C) and 56 controls with other neurological diseases of non-synuclein pathology. Aggregation of $\alpha$-syn was assessed by fluorescence of thioflavin T (ThT), a dye specific to the formation of amyloid fibrils. ThT fluorescence after PMCA was on average 2-4X reater in the CSF from patients with PD than patients with MSA. Based on this, the sensitivity for a diagnosis of PD and MSA was

Mitchell G. Miglis

muppidis@stanford.edu

Stanford Medical Center, Palo Alto, CA, USA

2 Stanford Neurosciences Health Center, 213 Quarry Road, 2nd Floor, Palo Alto, CA 94304, USA
93.6 and $84.6 \%$, respectively, with a specificity of $100 \%$. It was interesting to note that CSF samples from MSA patients aggregated faster but reached a lower plateau level than CSF from patients with PD. These findings were confirmed with brain tissue from post-mortem specimens of both PD and MSA patients, and indicate a more rapid and saturating seeding potential in MSA.

To analyze the biochemical differences between $\alpha$-syn aggregates of PD and MSA, the authors utilized spectroscopy to demonstrate that MSA aggregates have a higher proportion of $\beta$-sheet structure than PD aggregates. The authors took this analysis even further by performing d cryoelectron tomography on samples, which provides a threedimensional image of the fibrils. We encourage readers to review these images in the article, which are quite striking, and demonstrate, in the authors' words, "two protofilaments that intertwine in a left-handed helix with a diameter of around $9 \mathrm{~nm}$." They found that this helix differed in an obvious way between patients, with MSA patients exhibiting a greater number of twists in the helix, compared to the mostly straight PD fibrils. Finally, the authors explored the toxicity of aggregated $\alpha$-syn in cell culture and found that MSA-derived aggregates showed significant toxicity at concentrations $4 \mathrm{X}$ lower than those of PD-derived aggregates, indicating greater toxicity at lower doses.

In summary, this comprehensive and elegant study further supports the concept of prion-like propagation in the $\alpha$-synucleinopathies and supports the promise of RTQuIC as a non-invasive diagnostic and potentially prognostic biomarker. 


\section{Further support for CSF RT-QuIC in MSA}

While clinical diagnostic criteria have been developed to help improve the diagnosis of MSA, definitive MSA is only diagnosed post-mortem. Currently, MSA is diagnosed based on clinical evaluation and testing, such as autonomic testing or imaging studies, however, some of these specialized autonomic tests are not available at all centers. Additionally, there is no definitive biomarker for early MSA. To address these issues, Singer et al. recently published their work titled "Alpha-synuclein oligomers and neurofilament light chain in spinal fluid differentiate multiple system atrophy from Lewy body synucleinopathies" [2]. In this study, the authors obtained CSF from patients with early but clinically confirmed MSA, healthy matched controls, patients with PD, and dementia with Lewy bodies (DLB). The study was performed in two different cohorts, although the analysis included both groups. In the initial discovery cohort, they obtained CSF from 24 early MSA patients (diagnosed by a movement disorder specialist with supportive autonomic studies, and less than a score of 17 on the UMARS part 1 to ensure classification as early MSA) and 14 controls. Neurofilament light chain (NfL), a marker of axonal degeneration, was assessed in CSF by the ELISA method. PMCA was utilized to detect $\alpha$-synuclein oligomers in CSF. This was followed by a confirmatory, prospectively enrolled cohort of 38 patients with early MSA, 16 patients with PD, 13 patients with DLB, and 15 healthy controls.

Not surprisingly, NfLwas significantly elevated in MSA, when compared to healthy controls. PMCA to assess alpha-synuclein was absent in all controls and positive in all but two MSA patients. This was also positive in all patients with PD and all but two DLB samples. Surprisingly, the patterns and reaction kinetics of $\alpha$-synuclein oligomers from MSA patients were different from patients with PD and DLB. In PD and DLB, aggregation occurs later but with a maximum fluorescence of a higher level, allowing the differentiation of MSA (lower overall total immunofluorescence) compared to PD or DLB. Various cutoff values of $\mathrm{NfL}$ and immunofluorescence provide excellent sensitivity and specificity to difference MSA from PD/DLB and controls.

It not surprising that $\mathrm{NfL}$ levels were normal in healthy controls and highest in the rapidly progressive neurodegenerative syndrome of MSA compared to PD and DLB. The differing features of $\alpha$-synuclein aggregation and immunofluorescence in MSA and PD/DLB might help us understand the different clinical spectrum and rapid disease progression in MSA compared to other $\alpha$-synucleinopathies. While promising, PMCA CSF analysis failed to detect two patients in the MSA cohort, indicating less than ideal sensitivity. As clinicians, we hope that these CSF studies will soon be available commercially, as the diagnosis of MSA is often delayed. Furthermore, earlier diagnosis of MSA may lead to earlier recruitment in clinical trials, with the hope of more therapeutic interventions.

\section{Recumbent brain fog in postural tachycardia syndrome}

Cognitive symptoms, often described by patients as "brain fog", are one of the most debilitating symptoms of patients with postural tachycardia syndrome (POTS). Although most patients with POTS experience worsening of their symptoms on standing, many patients also report cognitive symptoms in the supine position [3]. In their recent publication "Cerebral blood flow and cognitive performance in postural tachycardia syndrome: Insights from sustained cognitive stress test," [4] Wells and colleagues attempted to understand whether prolonged cognitive stress reduces cerebral blood flow velocity in patients with POTS in a recumbent position. The authors enrolled 22 patients from their autonomic clinic who met the criteria for POTS during head-up tilt testing or within 10 min of active standing. All participants with POTS were allowed to stay on their treatment regimen, except for midodrine, which was held at least $15 \mathrm{~h}$ before testing. There was a control group consisting of 18 age- and sex-matched healthy subjects with no cardiac or autonomic symptoms.

Participants were seated in a climate-controlled facility $\left(22{ }^{\circ} \mathrm{C}\right)$ at a desk with back support to emulate normal working conditions. Their cerebral blood flow velocity (CBFv) was measured continuously with middle cerebral artery transcranial Doppler (TCD) ultrasound in the dominant hemisphere. Beat-to-beat heart rate and blood pressure, respiratory rate, and end-tidal carbon dioxide were measured continuously. Cognitive testing was performed to assess the domains of psychomotor speed and attention by measuring reaction time and rapid visual information processing, respectively. Both reaction time and rapid visual information processing were measured at baseline and after $30 \mathrm{~min}$ of prolonged cognitive stress testing (PCST), which was administered using the Cambridge ${ }^{\circledR}$ neuropsychological test automated battery. To assess acute symptoms, participants were given the orthostatic hypotension questionnaire with the Likert scale at baseline and after the prolonged cognitive stress testing. Participants were also given the quality of life assessment in MSA questionnaire (even though none of them had MSA) and the abbreviated composite autonomic symptom score-31 (COMPASS-31).

The authors found that at baseline, participants with POTS had higher resting heart rates than controls, however, mean resting $\mathrm{BP}$, respiratory rate, $\mathrm{ETCO}_{2}$, and $\mathrm{CBFv}$ did not 
differ between groups. After initial cognitive testing (ICT), the mean HR was consistently higher in patients with POTS. None of the other physiologic responses $\left(\mathrm{BP}, \mathrm{ETCO}_{2}\right.$, and $\mathrm{CBFv}$ ) differed between POTS and controls. When cognitive testing was performed after PCST, the HR response was similar between groups with consistent slowing as compared with during ICT. Following PCST, a greater reduction in $\mathrm{CBFv}$ was seen in the POTS group compared to controls. When repeated cognitive testing was performed following PCST, a longer delay in mean reaction time was seen in the POTS group, but not in the number of correct responses in the rapid visual information processing test. After participants were exposed to $5 \mathrm{~min}$ of orthostatic stress, the extent of change of physiologic parameters (HR, BP, $\mathrm{ETCO}_{2}$, and $\mathrm{CBFv}$ ) did not differ between groups from baseline to the end of 5-minute orthostatic stress. Orthostatic stress did result in significant increases in HR, systolic BP, diastolic $\mathrm{BP}$, and a significant decrease in CBFv in both groups.

Quality of life scores and autonomic symptom scores were significantly higher in the POTS group compared to the control group, reflecting a greater symptom burden. All patients with POTS described slowness of thinking and difficulty with concentration, while none of the 12 controls described these difficulties. The POTS group demonstrated consistently worse orthostatic symptoms, as expected.

Although the physiological basis of the brain fog phenomenon in POTS remains unclear, it is thought that brain fog may be related to decreased cerebral perfusion. Although previous studies in patients with POTS did not find a relationship between cognitive change and $\mathrm{CBFv}$ [5], there is evidence that oscillations in $\mathrm{CBFv}$ are associated with reduced neurovascular coupling and diminished cognitive performance in patients with POTS [6]. This is the first study to demonstrate that prolonged cognitive stress leads to reduced cerebral blood flow velocity in patients with POTS, which helps explain why patients with POTS experience brain fog in a recumbent position. Cerebral blood flow velocity did not differ in response to orthostatic stress between groups, which suggests that cognitive dysfunction in POTS patients cannot be explained soley by changes in cerebral blood flow.

A major limitation of this study is that, except for midodrine, participants remained on their cardiovascular medications. The authors did not control for vessel diameter, which affects the calculation of cerebral blood flow velocity, although the authors thought that vessel size variability was likely to be small. Nonetheless, this study provides evidence that prolonged cognitive stress leads to decreased cerebral blood flow velocity in patients with POTS and may account for some of the cognitive impairment experienced by patients.

\section{Compliance with ethical standards}

Conflict of interest The authors declare that they have no conflict of interest.

\section{References}

1. Shahnawaz M, Mukherjee A, Pritzkow S et al (2020) Discriminating $\alpha$-synuclein strains in Parkinson's disease and multiple system atrophy. Nature 578:273-277

2. Singer W, Schmeichel AM, Shahnawaz M et al (2020) Alphasynuclein oligomers and neurofilament light chain in spinal fluid differentiate multiple system atrophy from lewy body synucleinopathies. Ann Neurol 88:503-512

3. Ross AJ, Medow MS, Rowe PC, Stewart JM (2013) What is brain fog? An evaluation of the symptom in postural tachycardia syndrome. ClinAuton Res 23:305-311

4. Wells R, Malik V, Brooks AG et al (2020) Cerebral blood flow and cognitive performance in postural tachycardia syndrome: insights from sustained cognitive stress test. J Am Heart Assoc. https://doi. org/10.1161/JAHA.120.017861

5. Ocon AJ, Messer ZR, Medow MS, Stewart JM (2012) Increasing orthostatic stress impairs neurocognitive functioning in chronic fatigue syndrome with postural tachycardia syndrome. ClinSci. https://doi.org/10.1042/CS20110241

6. Stewart JM, Del Pozzi AT, Pandey A, Messer ZR, Terilli C, Medow MS (2015) Oscillatory cerebral blood flow is associated with impaired neurocognition and functional hyperemia in postural tachycardia syndrome during graded tilt. Hypertension. https ://doi.org/10.1161/HYPERTENSIONAHA.114.04576 